\title{
Detection and molecular characterization of infectious spleen and kidney necrosis virus from major ornamental fish breeding states in Peninsular Malaysia
}

\begin{abstract}
'Gold standard' OIE reference PCR assay was utilized to detect the presence of infectious spleen and kidney necrosis virus (ISKNV) in freshwater ornamental fish from Malaysia. From total of 210 ornamental fish samples representing 14 species, ISKNV was detected in 36 samples representing 5 fish species. All positive cases did not show any clinical signs of ISKNV. Three restriction enzymes analyses showed that the fish were infected by identical strains of the same virus species within Megalocytivirus genus. Major capsid protein (MCP) genes of 10 ISKNV strains were sequenced and compared with 9 other reference nucleotide sequences acquired from GenBank. Sequence analysis of MCP gene showed that all strains detected in this study were closely related to the reference ISKNV with nucleotide sequence identity that was ranging from $99.8 \%$ to $100 \%$. In addition, phylogenetic analysis of MCP gene revealed that viruses from genus Megalocytivirus can be divided into three genotypes: genotype 1 include reference ISKNV and all other strains that were detected in this study, genotype 2 include viruses closely related to red sea bream iridovirus (RSIV), and genotype 3 include viruses closely related turbot reddish body iridovirus (TRBIV).
\end{abstract}

Keyword: Infectious spleen and kidney necrosis virus; Major capsid protein gene; Megalocytivirus; Ornamental fish 